Case Study

\title{
Effect of pain scrambler therapy on shoulder joint pain and range of motion in patients who had undergone arthroscopic rotator cuff repair for the first time
}

\author{
Dong-Kyu Lee, PT, MS ${ }^{1,2)}$, Eun-Kyung KIM, PT, PhD ${ }^{3)^{*}}$ \\ 1) Department of Rehabilitation Science, Graduate School, Daegu University, Republic of Korea \\ 2) Department of Physical Therapy, Sunhan Hospital, Republic of Korea \\ 3) Department of Physical Therapy, Seonam University: 439 Chunhyang-ro, Namwon-si, \\ Jeollabuk-do 590-711, Republic of Korea
}

\begin{abstract}
Purpose] This study aimed to determine the effect of pain scrambler therapy on shoulder joint pain and range of motion in patients who had undergone arthroscopic rotator cuff repair for the first time. [Subjects and Methods] Pain scrambler therapy was administered once a day every 40 minutes for 10 days to patients that had undergone arthroscopic rotator cuff repair for the first time. The visual analog scale was used to measure pain, and a goniometer was used to measure shoulder range of motion. [Results] After 10 sessions of pain scrambler therapy, pain was significantly reduced from that before the treatment. In addition, shoulder range of motion was increased after 10 treatment sessions. [Conclusion] Thus, pain scrambler therapy greatly reduced pain and increased should range of motion in the patients who had undergone arthroscopic rotator cuff repair for the first time.

Key words: Pain scrambler treatment, Pain, Shoulder
\end{abstract}

(This article was submitted Jan. 12, 2016, and was accepted Apr. 7, 2016)

\section{INTRODUCTION}

Shoulder pain has an incidence of $7-36 \%$ and is a common symptom of musculoskeletal disease, which occurs in $20 \%$ of all adults once in their lifetimes ${ }^{1)}$. Shoulder pain may be caused by myofascial pain syndrome, disruption of the rotator cuff, frozen shoulder, shoulder impingement syndrome, and damaged ligament ${ }^{1,2)}$. The most frequently reported cause is disruption of the rotator cuff ${ }^{2}$. As the rotator cuff contributes more to the stability than to the motion of the shoulder joint, disruption occurs because of conditions such as degenerative change, instability of blood circulation, calcific tendinitis, tissue alteration, and repetitive activity ${ }^{3}$.

Patients with impaired rotator cuffs consider surgical treatment if pain continues despite consistent drug intake, electrical stimulation, therapeutic exercise, or lifestyle change ${ }^{4,5}$. The purpose of surgical treatment for patients with impaired rotator cuff is to improve quality of life by improving shoulder joint function and reducing pain ${ }^{6}$. However, surgical treatment always has potential side effects such as failure or infection. In some cases, pain may not disappear but persist, or stiffened joints may prevent recovery ${ }^{6,7)}$.

Pain scrambler is not just a general treatment but also a new type of pain treatment device that restores impaired painrecognition nerve system to normal by training the transmission of non-pain and eliminating pain ${ }^{8,9)}$. Pain scrambler therapy has been reported to have an effect on chronic, rare, postsurgical, neuropathic, and muscular pains ${ }^{8-11)}$.

This study aimed to determine the effect of pain scrambler therapy on shoulder joint pain and range of motion in patients who had undergone arthroscopic rotator cuff repair for the first time.

\footnotetext{
*Corresponding author. Eun-Kyung Kim (E-mail: kek74ing@naver.com)

(C)2016 The Society of Physical Therapy Science. Published by IPEC Inc.

This is an open-access article distributed under the terms of the Creative Commons Attribution Non-Commercial No Derivatives (by-nc-nd) License $<$ http://creativecommons.org/licenses/by-nc-nd/4.0/>.
} 


\section{SUBJECTS AND METHODS}

In this study, patients who had undergone arthroscopic rotator cuff repair for the first time were examined. The patients' general characteristics (mean values) were as follows: age, 60 years; weight, $53 \mathrm{~kg}$; and height, $161 \mathrm{~cm}$. The patients had undergone arthroscopic rotator cuff repair for the first time, with no other problems regarding blood pressure, pulse, breathing, consciousness, or sensation. Before proceeding with the research, the purpose and method of the research was explained to the patients and consent was obtained. All the subjects provided written informed consent prior to participation in the study according to the ethical standards of the Declaration of Helsinki.

Pain scrambler therapy was performed by using a special type of electrode with five channels. The special electrode was attached $4 \mathrm{~cm}$ away from the most painful areas. The frequency was $43-52 \mathrm{~Hz}$, and the strength of the stimulation was $5 \mathrm{~mA}$, which was quite harmless and was used to transmit a natural electric signal. The waveform of non-pain information in the pain scrambler was composed of 16 waveforms. The subjects received the treatment once a day every 40 minutes for 10 days.

Pain was measured using the visual analog scale (VAS). VAS is a commonly used instrument for measuring pain intensity. With this method, the pain level was visualized and the subjects were asked to determine the intensity of pain by using a 10-pointscale. No pain was scored 0 , and unbearable pain was scored as 10 .

Shoulder joint range of motion was measured by using a goniometer. In addition, flexion, abduction, and external rotation were measured. Flexion of the shoulder joint was measured while the subject was lying down. The axis of the goniometer was fixed to the acromion process. The stationary arm was set horizontal to the central line of the trunk; and the moving arm, to the central line of the upper arm. The angle when the subjects curved their arms forward as much as possible was measured. Shoulder joint abduction was measured while the subjects were lying down so that the trunk would not move. The axis of the goniometer was set to the acromion process, and the movable element was set to the central line of the upper arm. The angle when the subjects spread their arms aside as much as possible was measured. Moreover, the subjects were asked to curve their arms to $90^{\circ}$ and again spread them aside to $90^{\circ}$ while lying down for measuring the external rotation of the shoulder joints. The axis of the goniometer was set to the elbow, and the stationary arm was fixed vertical to the floor. The angle was measured when the subjects raised their lower arms as much as possible without moving the elbow. Shoulder range of motion and pain were measured before treatment and after completion of 10 sessions of pain scrambler therapy.

\section{RESULTS}

Pain was measured by using the VAS. Although the VAS score was 8 points before pain scrambler therapy was initiated, it increased by 1 point after 10 treatment sessions, indicating that pain was reduced. Flexion, abduction, and external rotation were measured for determining shoulder joint range of motion. Before pain scrambler therapy was started, the flexion angle was $101^{\circ}$. However, after 10 treatment sessions, it increased to $152^{\circ}$. Similarly, before initiation of pain scrambler therapy, abduction and external rotation were respectively $94^{\circ}$ and $19^{\circ}$ but increased to $148^{\circ}$ and $25^{\circ}$ after 10 treatment sessions.

\section{DISCUSSION}

This study aimed to determine the effect of pain scrambler therapy on shoulder joint pain and range of motion in patients who had undergone arthroscopic rotator cuff repair for the first time. The results showed that pain decreased and shoulder joint range of motion increased after pain scrambler therapy.

Pain scrambler therapy involves pain recognition as single information based on the information theory and to encode non-pain information artificially for transmitting it to the brain through A-delta and C-fibers, which are nerve pathways for pain ${ }^{8)}$. Moreover, it is a method whereby the autonomic nerve controlling function of the brain can be restored through nerve pathways for pain by encoding non-pain information ${ }^{8,9)}$. Based on 16 non-pain waveforms produced artificially by the pain scrambler, artificial neuron information can be printed and transmitted in the form of wave signals such as $43-52 \mathrm{~Hz}$, with $5 \mathrm{~mA}$ as maximum, through the body and then to the pain-inducing nerve ${ }^{8,9)}$. Through this method, the brain is able to autonomously recover neuroregulation and improve several conditions such as chronic, incurable, cancerous, and neurotic pains $^{8-11)}$. Commonly used methods such as drug intake, injection, and surgical treatment block the pathway whereby pain enters the central nervous system and is transmitted to the brain, thus preventing patients from feeling pain by stimulating A-beta nerves ${ }^{9,10)}$. However, the pain scrambler applies the non-pain signal naturally to areas where pain is felt and does not block thepathway ${ }^{8-10)}$. Synthesizing and applying these theories would have a positive effect on pain reduction in patients who have undergone arthroscopic rotator cuff repair for the first time. This approach would contribute to the recovery of shoulder joint range of motion.

As this study examined only a few subjects, it is difficult to generalize its research results. Furthermore, the long-term effects of pain scrambler therapy could not be investigated. To generalize the results of this research, more long-term research and follow-up studies should be conducted in patients who have undergone arthroscopic rotator cuff repair for the first time. 


\section{REFERENCES}

1) Green S, Buchbinder R, Glazier R, et al.: Systematic review of randomised controlled trials of interventions for painful shoulder: selection criteria, outcome assessment, and efficacy. BMJ, 1998, 316: 354-360. [Medline] [CrossRef]

2) Ostör AJ, Richards CA, Prevost AT, et al.: Diagnosis and relation to general health of shoulder disorders presenting to primary care. Rheumatology (Oxford), 2005, 44: 800-805. [Medline] [CrossRef]

3) Kieft GJ, Bloem JL, Rozing PM, et al.: Rotator cuff impingement syndrome: MR imaging. Radiology, 1988, 166: 211-214. [Medline] [CrossRef]

4) Wirth MA, Basamania C, Rockwood CA Jr: Nonoperative management of full-thickness tears of the rotator cuff. Orthop Clin North Am, 1997, 28: 59-67. [Medline] [CrossRef]

5) Jung JJ, Cho KH, Yu JH: Effects of scapular stabilizing exercise in patients with partial-thickness rotator cuff tear. J Phys Ther Sci, 2012, 24: 1173-1175. [CrossRef]

6) Baysal D, Balyk R, Otto D, et al.: Functional outcome and health-related quality of life after surgical repair of full-thickness rotator cuff tear using a mini-open technique. Am J Sports Med, 2005, 33: 1346-1355. [Medline] [CrossRef]

7) Flatow EL, Cordasco FA, Bigliani LU: Arthroscopic resection of the outer end of the clavicle from a superior approach: a critical, quantitative, radiographic assessment of bone removal. Arthroscopy, 1992, 8: 55-64. [Medline] [CrossRef]

8) Sabato AF, Marineo G, Gatti A: Scrambler therapy. Minerva Anestesiol, 2005, 71: 479-482. [Medline]

9) Marineo G: Untreatable pain resulting from abdominal cancer: new hope from biophysics? JOP, 2003, 4: 1-10. [Medline]

10) Smith TJ, Coyne PJ, Parker GL, et al.: Pilot trial of a patient-specific cutaneous electrostimulation device (MC5-A Calmare $\left.{ }^{\circledR}\right)$ for chemotherapy-induced peripheral neuropathy. J Pain Symptom Manage, 2010, 40: 883-891. [Medline] [CrossRef]

11) Pachman DR, Weisbrod BL, Seisler DK, et al.: Pilot evaluation of Scrambler therapy for the treatment of chemotherapy-induced peripheral neuropathy. Support Care Cancer, 2015, 23: 943-951. [Medline] [CrossRef] 